\title{
Additive-Manufacturing of 3D Glass-Ceramics down to Nanoscale Resolution
}

\section{Darius Gailevičius ${ }^{1,2, *}$, Viktorija Padolskytè ${ }^{1}$, Lina Mikoliūnaitè ${ }^{3}$, Simas Šakirzanovas ${ }^{3}$, Saulius Juodkazis ${ }^{4,5, *}$, and Mangirdas Malinauskas ${ }^{1, *}$}

${ }^{1}$ Laser Research Center at Vilnius University, Sauletekio Ave. 10, Vilnius, LT-10223, Lithuania

${ }^{2}$ Femtika Ltd., Saulètekio Ave. 15, Vilnius, LT-10224, Lithuania

${ }^{3}$ Faculty of Chemistry and Geosciences, Vilnius University, Naugarduko Str. 24, Vilnius, LT-03225, Lithuania

${ }^{4}$ Swinburne University of Technology, John St., Hawthorn 3122 Vic, Australia

${ }^{5}$ Melbourne Centre for Nanofabrication, the Victorian Node of the Australian National Fabrication Facility, 151

Wellington Rd., Clayton 3168 Vic, Australia

${ }^{*}$ Correspondence: darius。gailevicius $\Theta f f \circ s_{\circ} t u d_{\circ} v_{\circ}$ olt

*Correspondence: sjuodkazis $\Theta f f_{\circ}$ swin $_{\circ}$ edu。au

*Correspondence: mangirdas。malinauskas $\Theta f f_{\circ} v_{\circ}$ 。t

\begin{abstract}
Fabrication of a true-3D inorganic ceramic with resolution down to nanoscale using sol-gel resist precursor is demonstrated. The method has an unrestricted free-form capability, control of the fill-factor, and high fabrication throughput. A systematic study of the proposed approach based on ultrafast laser 3D lithography of organic-inorganic hybrid sol-gel resin followed by a heat treatment enabled formation of inorganic amorphous and crystalline composites guided by the composition of the initial resin. The achieved resolution of $100 \mathrm{~nm}$ was obtained for 3D patterns of complex free-form architectures. Fabrication throughput of $50 \times 10^{3}$ voxels/s is achieved; voxel - a single volume element was recorded by a single pulse exposure. After a subsequent thermal treatment, ceramic phase was formed depending on the temperature and duration of the heat treatment as validated by Raman micro-spectroscopy. The X-ray diffraction (XRD) revealed a gradual emergence of the crystalline phases at higher temperatures with a signature of cristobalite $\mathrm{SiO}_{2}$, a high-temperature polymorph. Also, the tetragonal $\mathrm{ZrO}_{2}$ phase known for its high fracture strength was observed. This 3D nano-sintering technique is scalable from nano- to millimeter dimensions and opens a conceptually novel route for optical 3D nano-printing of various crystalline inorganic materials defined by an initial composition for diverse applications for microdevices in harsh physical and chemical environments and high temperatures.
\end{abstract}

Keywords: Additive-Manufacturing, 3D Printing, Glass-Ceramics, Nanoscale, Laser 3D lithography, SZ2080, Cristobalite, Zirconia, Nanocomposites, Calcination.

\section{Introduction}

We show that a popular hybrid organic-inorganic sol-gel resist SZ2080 can be converted into a material with entirely different properties obtained via polymer-to-ceramic transition guided by a high temperature sintering and oxidation. The silica and zirconia precursors present in the resist in the $\sim 20 \%$ mass of inorganic component will lead to emergence of silica and zirconia crystalline phases in the final sintered ceramic material. Importantly, a proportional downscaling of the 3D polymerized object takes place with significant volume change of 40-50\% dependent on annealing protocol without distortion of the proportions of the initial 3D design. The temperature-guided resizing and the composition change can be potentially tailored to form 3D free-form patterns of complexity which is not amenable by other micro-/nano-fabrication methods.

In this study, we explore a possibility to apply ultrafast 3D laser nanolithography ${ }^{1}$ in conjunction with heat-treatment ${ }^{2}$ to acquire ceramic 3D structures in micro- and nano-scale. Laser fabrication (Fig. 1) allows production of initial 3D structures with relatively small (hundreds-of-nm) feature sizes out of hybrid organic-inorganic material SZ2080 ${ }^{3}$. Then, a post-fabrication sintering at different temperatures up to $1500^{\circ} \mathrm{C}$ in the air atmosphere facilitates decomposition of organic part (80\%) and results in the glass-ceramic hybrid material. Resolution in the final 3D structure is superior to that of the as-fabricated structures. Both, filled volume as well as complex free-form 3D objects can be resized and their structural composition changed.

Ultrafast lasers are extending their applications towards advanced nanoscale processing of materials ${ }^{4,5}$. Recently, additive manufacturing of 3D micro-/nano-structures followed by heat-treatment protocols for downscaling their dimensions while keeping their initial geometry was reported ${ }^{6}$, yet the attention was not paid in experimentally validated explanation of material conversion process $^{7}$, thus the potential advantages besides resizing were not investigated ${ }^{8,9}$. Alternatively, the physical addition 


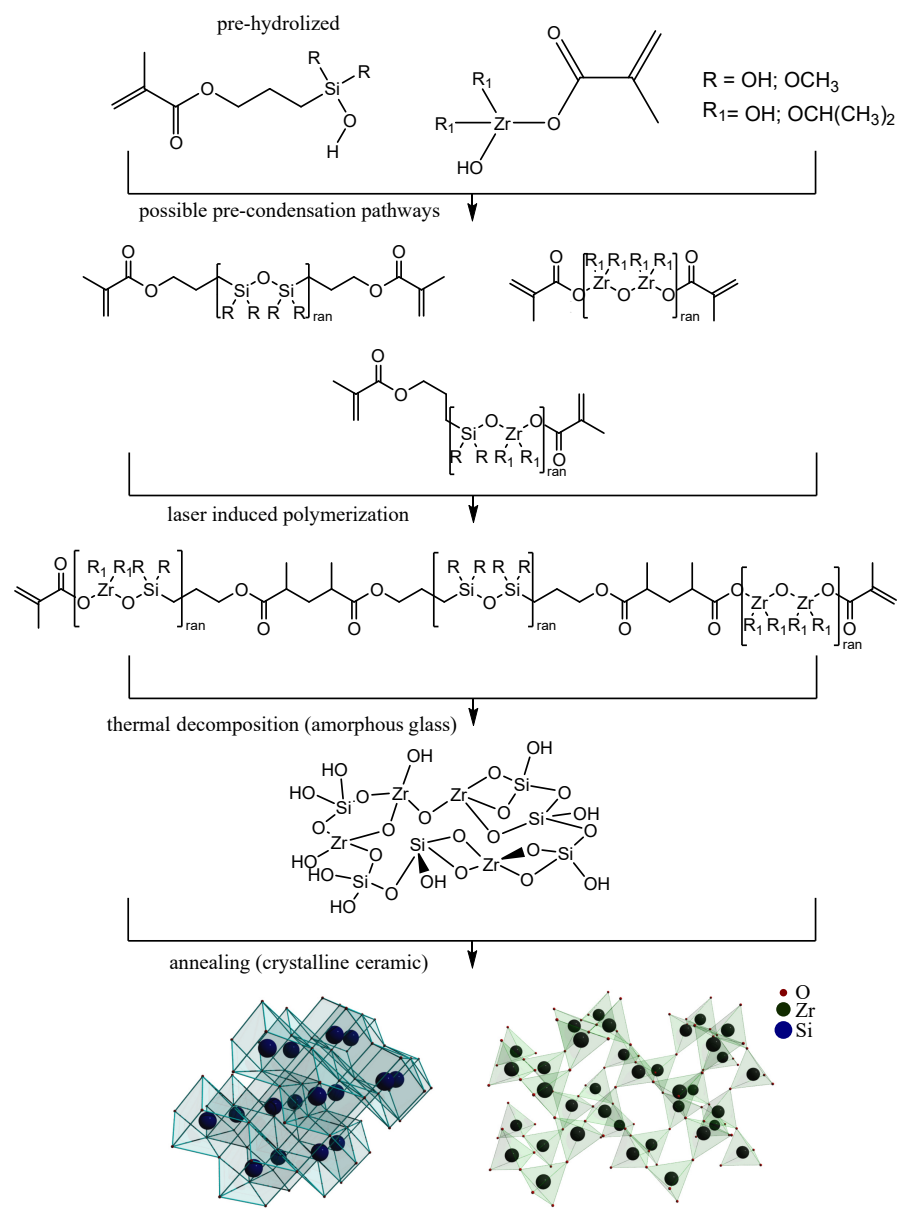

Figure 1. Illustration of the main steps in synthesis of ceramics out of hybrid SZ2080 followed from laser induced polymerization that occurs during direct laser writing. During first stage of calcination, organic part is removed from the matrix and an inorganic glass matrix forms. As temperature is increased further, crystallization occurs and polycrystalline ceramic phase forms. Crystal structure of cristobalite and $\mathrm{t}-\mathrm{ZrO}_{2}$ are shown in bottom row.

of metal nanoparticles resulted in roughening of the structures after turning them fully into inorganic coatings ${ }^{10}$. This limits their applications for functional 3D nanostructures where pure inorganic materials and/or optical quality and structural uniformity of the patterns and workpieces are required ${ }^{11}$. Lastly, up to know there is no report on true-3D ceramic ${ }^{12}$ or glass ${ }^{13,14}$ structures with higher than tens-of-micrometers resolution despite specially designed pre-ceramic resins ${ }^{15}$.

Here, we show a straight-forward method to make 3D glass and ceramic structures with resolution down to the nanoscale with final composition tailored by the initial materials.

\section{Experimental}

To prove this we used the prepolymer synthesized as described $\mathrm{in}^{3}$, yet we do not use any photonitiator. We made 3D laser direct writen structures from this material and performed heat-treatment in temperature range of $1000-1500^{\circ} \mathrm{C}$ and analyzed the changes in Raman spectra and also performed X-ray diffraction measurements (XRD).

More specifically, for our experiment, we fabricated 3D structures with different geometries. These included: bulk - cubes, periodic - 3D woodpile micro lattices, free form structures - micro-sculptures, combining bulk and nanometer feature elements with complex bends, and macroscopic hexagonal 3D lattices which are usually used as artificial cell scaffolds. For laser structuring, we used a $300 \mathrm{fs}$ pulse duration $515 \mathrm{~nm}$ wavelength $200 \mathrm{kHz}$ pulsed laser beam focused through different numerical value objectives lenses, which are in the range of $0.8 \mathrm{NA}$ and $1.4 \mathrm{NA}$. Other relevant fabrication parameters were tuned to be approximately near the middle of the fabrication window ${ }^{16}$. We developed the resulting structures in methyl-isobutyl-ketone for one hour and treated them with a Piranha solution to remove organic contaminants from their surface. The geometrical changes and stability were tracked by making optical images and scanning electron microscope (SEM) micrographs. 

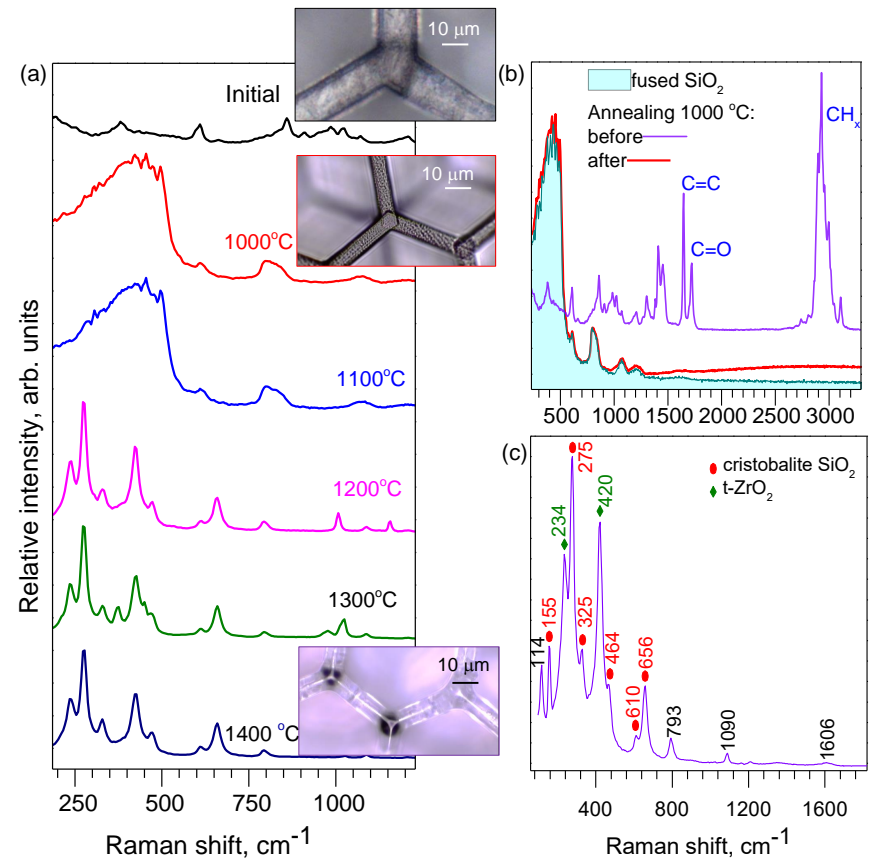

Figure 2. Raman spectrum of laser structured SZ2080 before and after heat-treatment (for $1 \mathrm{~h}$ in air). Disappearance of sharp peaks represents the changed make-up of the material after heat-treatment. (a) Change of Raman spectrum at several annealing temperatures; optical images of 3D-micro scaffolds $\left(\mathrm{T}=1000,1400^{\circ} \mathrm{C}\right)$ from the sites where Raman spectra were measured. (b) Detailed spectrum of the initial structure and heat treated structure at $\mathrm{T}=1000^{\circ} \mathrm{C}$ in comparison to the fused silica. (c) Raman spectrum after the highest temperature $\mathrm{T}=1400^{\circ} \mathrm{C}$ annealing with peak matching to the cristobalite and $\mathrm{t}-\mathrm{ZrO}_{2}$.

For the (micro-)Raman spectra analysis we choose our hexagonal scaffolds ${ }^{17}$ that due their millimeter scale are easy to handle. We measured micro-Raman spectra on the beams and intersection of hexagonal scaffolds in each relevant step: before heat treatment, after heat treatment at different temperatures $\mathrm{T} \leq 1500^{\circ} \mathrm{C}$. To corroborate and interpret the results we also performed an XRD analysis on calcinated and then powdered SZ2080.

\section{Results}

The micro-Raman measurements are summarized in Fig. 2. As the temperature increases the spectral shape changes and evolves via qualitatively two distinct form-factors (Fig. 2). Close examination of the initial spectrum and comparison to that for $\mathrm{T}=1000^{\circ} \mathrm{C}$ reveals that they differ by the molecular vibrations which can be associated with the carbon-carbon, carbon-oxygen, carbon-hydrogen bonds. After heat-treatment those spectral lines vanishes (Fig. 2b). The new spectral form coincides with that typical for silica glass; we measured a control sample of fused silica.

Increasing the temperature further in steps of $\Delta \mathrm{T}=100^{\circ} \mathrm{C}$ results in a new qualitative change of spectrum at $\mathrm{T}=1200^{\circ} \mathrm{C}$. More pronounced peaks emerge with increased temperature. At the highest temperature (Fig. 2c), a few pronounced peaks become apparent. They coincide with vibrational signatures of the cristobalite ${ }^{18}$ and tetragonal zirconia ${ }^{19}$ phases, which are formed at high temperatures ${ }^{20,21}$.

Figure 3 shows X-ray diffraction (XRD) data of annealed powder samples. At the lowest treatment temperature $\mathrm{T}=1000^{\circ} \mathrm{C}$, broad peaks indicate formation of a glassy amorphous phase and only initial seeds of a crystalline phase. Also, the high background of XRD signal confirms a substantial amount of amorphous material. As the temperature is increased, the peaks become exceedingly pronounced distinguishably showing an increasing dominance of the crystalline phase. The XRD peaks match well with reference data for cristobalite 22 and tetragonal zirconia ${ }^{23}$, proving that annealed material is a mix of inorganic crystalline phases. From the XRD pattern, the period $d$ which corresponds to the most pronounced peaks at the diffraction angle $2 \theta$, given by the Bragg's condition $d=\lambda /(2 \sin \theta)$ can be estimated. The size $L$ of the nano-crystalline phase follows from the Scherrer's equation $L=K \lambda /(B(2 \theta) \cos \theta$; where $K=0.89$ for spherical crystals and $B(2 \theta)$ [rad] is the peak's angular bandwidth at full width half maximum and the $\mathrm{Cu} \mathrm{K}_{\alpha}$ emission at $\lambda=1.5406 \AA$ was used. The most pronounced peaks are observed after treatment at $\mathrm{T}=1500^{\circ} \mathrm{C}$ at Bragg diffraction angle $2 \Theta \approx 30.14^{\circ}$ corresponds to the crystallite size of $L \simeq 117.5 \mathrm{~nm}$ for the $\mathrm{t}-\mathrm{ZrO}_{2}$ and similarly at the angle $2 \Theta \approx 21.82^{\circ}$ the size of cristobalite nano-crystallites was $L \simeq 30.8 \mathrm{~nm}$. 


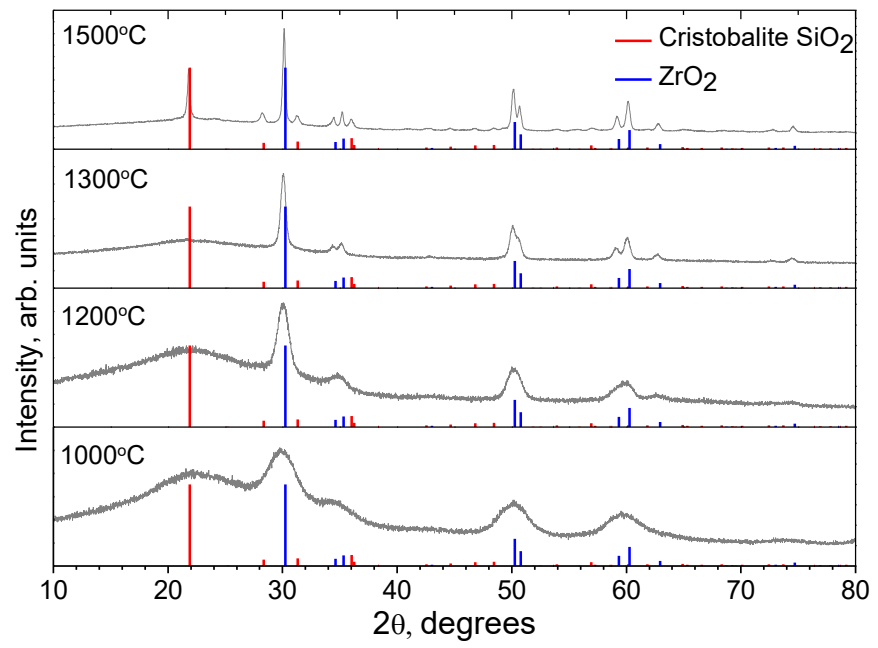

Figure 3. X-ray diffraction (XRD) analysis of SZ2080 resist heat treated at different temperatures for 1 hours in air in ambient pressure. Broad peaks become more pronounced and evolve with temperature into sharp signature peaks of the two known crystalline phases of silica and zirconia.

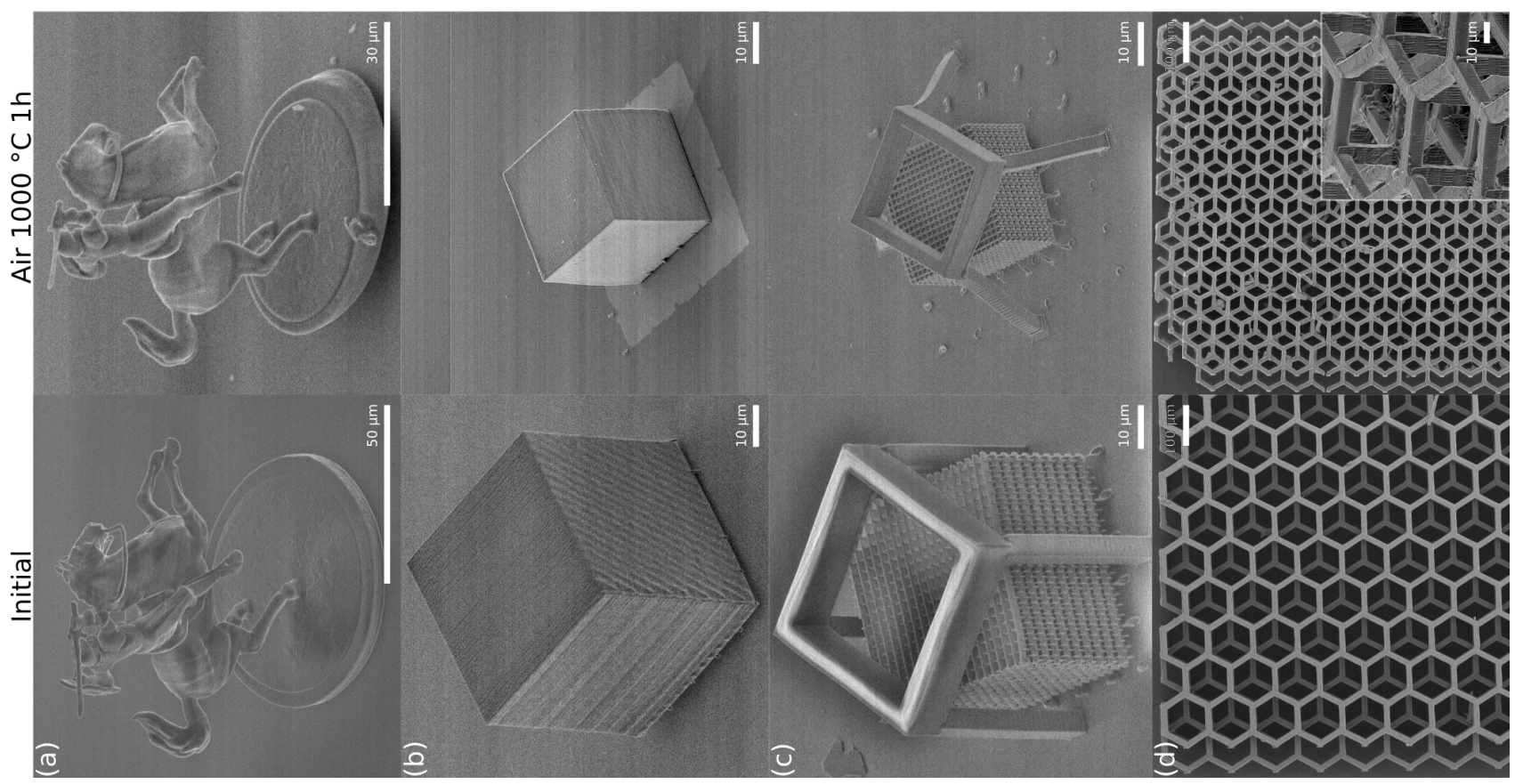

Figure 4. Micro-graphs of different initial and treated structures $\left(1000^{\circ} \mathrm{C}\right.$ for $\left.2 \mathrm{~h}\right)$. Down-sizing of solid volumetric and free-form structures (with correspondingly high and low initial volume fractions of polymer). From top to bottom: (a) a free-form sculpture Vytis (Coat of arms of Lithuania), (b) homogeneous cube structure, (c) photonic crystal (periodic) structure with cage and (d) hexagonal scaffold.

A size evolution of the $\mathrm{t}-\mathrm{ZrO}_{2}$ crystallites traced by the most prominent $\langle 111\rangle \mathrm{XRD}$ peak was $L \approx 1,14,118 \mathrm{~nm}$ as temperature was increasing trough $1000,1200,1500^{\circ} \mathrm{C}$, respectively.

As for the geometrical stability of the structures, they remain stable at least up to $\mathrm{T} \leq 1200^{\circ} \mathrm{C}$. After which, the structures appear to show signs of melting and deformation. Sharp features become noticeably rounded by surface tension of the molten phase. We show that for the primary "glassy" phase the structures retain their shape without shape distortions (Fig. 4). The effect of much higher temperatures is shown in the optical images in Fig. 2a. 


\section{Conclusions and Outlook}

It is shown that by a high-temperature calcination of 3D polymerized structures, initially made by $3 \mathrm{D}$ laser writing in the organic-inorganic SZ2080 polymer resist produces either silica-based glass or a polycrystalline ceramic pure inorganic material. Glass phase dominate in the samples annealed at moderate temperatures up to $\sim 1200^{\circ} \mathrm{C}$. When samples were annealed above $\sim 1200^{\circ} \mathrm{C}$, formation of the polycrystalline silica and zirconia was observed, in particular, the cristobalite and t-zirconia phases.

The presented modifications of silica-zirconia-rich resist SZ2080 from glass to polycrystalline ceramic by annealing shows a principle of the thermally guided 3D material printing which has nanoscale resolution. Isotropic down-sizing of the initial 3D polymerized objects with a volume fraction of 0.5-to-1 simplifies fabrication since there is no need to alter proportions of the initial material as it is widely used in DLW 3D nanolithography of photonic crystals, micro-optics and biomedical scaffolds in order to eliminate the effect of anisotropic shrinkage.

One can foresee a possibility to create required polymerizable mixtures which will lead to the final compounds of tailored composition - stoichiometry and size of polycrystalline phases according to the phase diagram ${ }^{24,25}$. Mechanical and chemical properties of the final structures and objects will acquire new features, especially resilience at harsh physical and chemical environments. Since nanoscale materials can initiate precipitation and guide growth of nano-crystallites, a wide field for experimentation horizons are widened by the presented modality of additive manufacturing.

\section{Conflicts of interest}

There are no conflicts to declare.

\section{Acknowledgements}

US AMRDEC grant No. W911NF-16-2-0069 "Enhanced Absorption in Stopped-Light Photonic Nanostructures: Applications to Efficient Sensing" project is acknowledged for the financial support. SJ is grateful for the partial support via ARC DP170100131 grant.

\section{References}

1. Malinauskas, M. et al. Ultrafast laser processing of materials: from science to industry. Light. Sci. Appl. 5, e16133-e16133 (2016).

2. Jonusauskas, L. et al. Optically clear and resilient free-form $\mu$-optics 3D-printed via ultrafast laser lithography. Materials 10, 12 (2017).

3. Ovsianikov, A. et al. Ultra-low shrinkage hybrid photosensitive material for two-photon polymerization microfabrication. ACS Nano 2 , 2257-2262 (2008).

4. Wang, H. et al. Photodynamic assembly of nanoparticles towards designable patterning. Nanoscale Horiz. 1, 201-211 (2016).

5. Ding, H., Zhang, Q., Gu, Z. \& Gu, M. 3d computer-aided nanoprinting for solid-state nanopores. Nanoscale Horiz. 3, 312-316 (2018).

6. Seniutinas, G. et al. Beyond $100 \mathrm{~nm}$ resolution in 3D laser lithography - post processing solutions. Microelectron. Eng. 191, 25-31 (2018).

7. Bauer, J., Schroer, A., Schwaiger, R. \& Kraft, O. Approaching theoretical strength in glassy carbon nanolattices. Nat. Mater. 15, 438-443 (2016).

8. Li, J., Jia, B. \& Gu, M. Engineering stop gaps of inorganic-organic polymeric 3 d woodpile photonic crystals with post-thermal treatment. Opt. Express 16, 20073-20080 (2008).

9. Zakhurdaeva, A. et al. Custom-designed glassy carbon tips for atomic force microscopy. Micromachines 8, 285 (2017).

10. Vyatskikh, A. et al. Additive manufacturing of 3D nano-architected metals. Nat. Commun. 9, 593 (2018).

11. Pham, T. et al. Three-dimensional sicn ceramic microstructures via nano-stereolithography of inorganic polymer photoresists. Adv. Func. Mater. 16, 1235-1241 (2006).

12. Eckel, Z. C. et al. Additive manufacturing of polymer-derived ceramics. Science 351, 58-62 (2015).

13. Kotz, F. et al. Glassomer-processing fused silica glass like a polymer. Adv. Mater. 30, 1707100 (2018).

14. Kotz, F. et al. Three-dimensional printing of transparent fused silica glass. Nature 544, 337-339 (2017).

15. Zocca, A. et al. Sioc ceramics with ordered porosity by 3d-printing of a preceramic polymer. J. Mater. Res. 28, 2243-2252 (2013).

16. Žukauskas, A. et al. Tuning the refractive index in $3 \mathrm{~d}$ direct laser writing lithography: towards grin microoptics. Laser Photon. Rev. 9, 706-712 (2018).

17. Mačiulaitis, J. et al. Preclinical study of sz2080 material 3d microstructured scaffolds for cartilage tissue engineering made by femtosecond direct laser writing lithography. Biofabrication 7, 015015 (2015).

18. Ilieva, A., Mihailova, B., Tsintsov, Z. \& Petrov, O. Structural state of microcrystalline opals: A raman spectroscopic study. Amer. Mineral. 92, 1325-1333 (2007). 
19. Barberis, P., Merle-Méjean, T. \& Quintard, P. On raman spectroscopy of zirconium oxide films. J. Nucl. Mater. 246, 232-243 (1997).

20. Černok, A. et al. Compressional pathways of $\alpha$-cristobalite, structure of cristobalite xi, and towards the understanding of seifertite formation. Nat. Commun. 8, 15647 (2017).

21. Vasanthavel, S., Derby, B. \& Kannan, S. Stabilization of a t-zro2 polymorph in a glassy sio2 matrix at elevated temperatures accomplished by ceria additions. Dalton Trans. 46, 6884-6893 (2017).

22. Pluth, J., Smith, J. \& Faber Jr, J. Crystal structure of low cristobalite at 10, 293, and 473 k: Variation of framework geometry with temperature. J. Appl. Phys. 57, 1045-1049 (1985).

23. Bouvier, P., Djurado, E., Lucazeau, G. \& Le Bihan, T. High-pressure structural evolution of undoped tetragonal nanocrystalline zirconia. Phys. Rev. B 62, 8731 (2000).

24. Curtis, C. \& Sowman, H. Investigation of the thermal dissociation, reassociation, and synthesis of zircon. J. Amer. Ceram. Soc. 36, 190-198 (1953).

25. Telle, R., Greffrath, F. \& Prieler, R. Direct observation of the liquid miscibility gap in the zirconia-silica system. J. Eur. Ceram. Soc. 35, 3995-4004 (2015).

26. Lin, J. et al. On-chip three-dimensional high-q microcavities fabricated by femtosecond laser direct writing. Opt. Express 20, 10212-10217 (2012).

27. Drs, J., Kishi, T. \& Bellouard, Y. Laser-assisted morphing of complex three dimensional objects. Opt. express 23, 17355-17366 (2015). 


\section{Supplement}

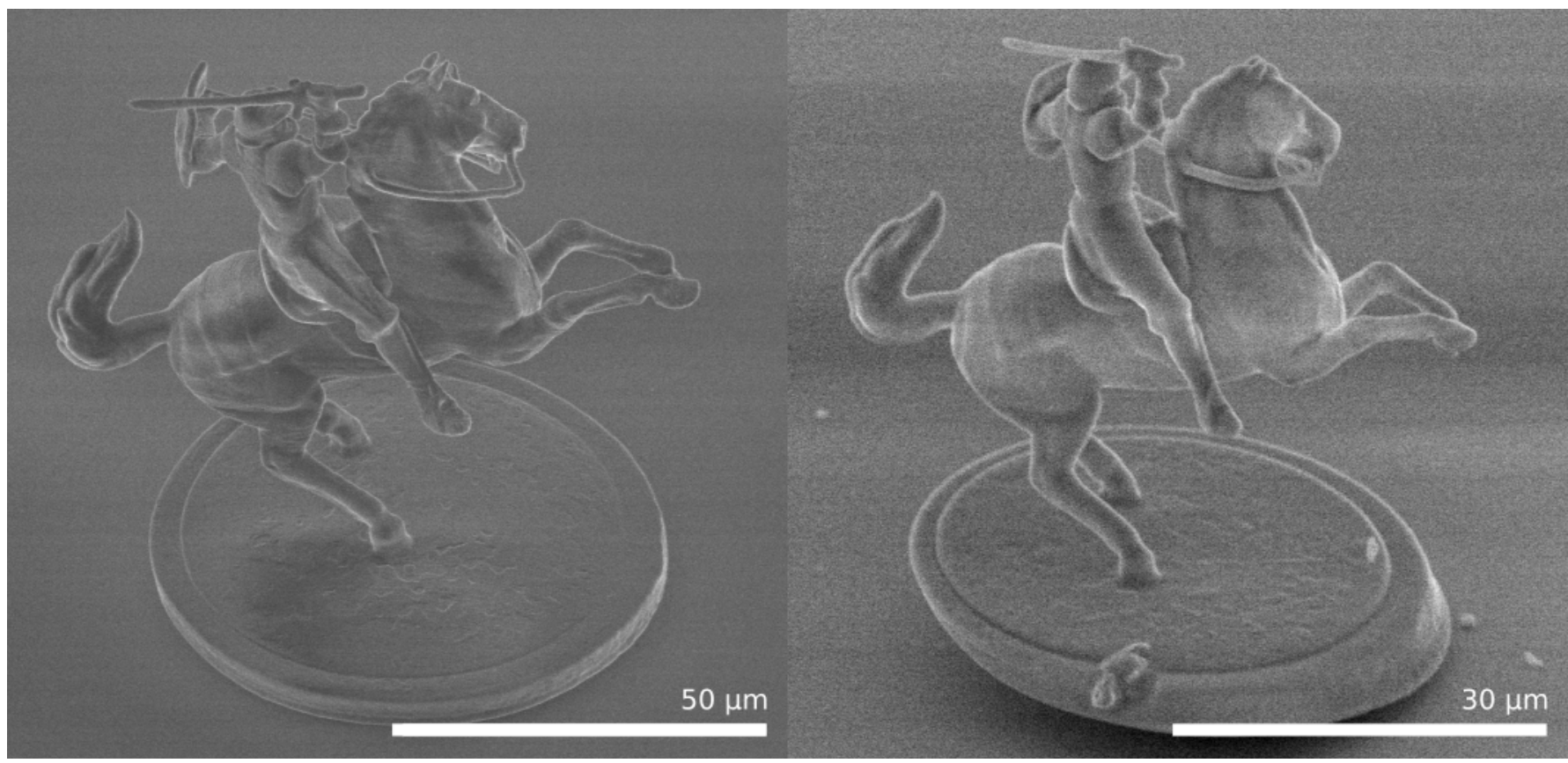

Figure 1. Uniform 3D down-scaling by 3D nano-sintering. SEM micro-graph of a ceramic micro-sculpture after sintering at $1200^{\circ} \mathrm{C}$ for one hour (right). Initial material SZ2080 resist (all dimensions were $1.7^{\times}$larger; note the different scale bars).

\section{Preparation of SZ2080 powder}

A large (macroscopic) amount of material was required for XRD analysis therefore the pulverized samples were prepared by the described method.

While the pre-polymer is usually prepared by starting with the condensation reaction of the metallo-organic part by heating to temperature no more than $100{ }^{\circ} \mathrm{C}$ for a few hours, the powder sol-gel precursor for analysis had to be made as a dry sample. Therefore, heating for $70{ }^{\circ} \mathrm{C}$ for 72 hours was performed. It was confirmed that the sample had not been polymerized by its dissolution when immersed in the pure iso-butyl-ketone developer for one hour.

The dried resist was ground up to a fine powder and exposed using the fourth harmonic of an Nd:YAG laser. The parameters were as follows: wavelength was $\lambda=266 \mathrm{~nm}$, pulse duration $-\tau \approx 5 \mathrm{~ns}$, pulse energy $-E_{\text {imp }} \approx 25 \mathrm{~mJ}$, pulse repetition rate $f=2 \mathrm{~Hz}$, the beam intensity profile was a flat-top with diameter of $\sim 1$ inch. The powder was laser exposed for 6 hours while being mechanically mixed; the dry amount of powder was approximately $300 \mathrm{mg}$. The mixture was developed in a 2-propanol bath while being agitated for 48 hours. After filtering out the solid polymer particles, we confirmed that polymerization had occurred by observing that it was not dissolved in iso-butyl-ketone when immersed for 1 hour.

After drying the powder at room conditions for 7 days, heat-treatment was performed at the indicated temperatures in corundum crucibles. 1

\section{Geometric changes accompanying formation of the ceramic phase}

Here we discuss transformation of geometry of the structure during transformation to the ceramic state as the temperature is increased. The strongest changes occur after the threshold of $1100^{\circ} \mathrm{C}<T$.

A short qualitative analysis of geometrical changes for a selected treatment temperature of $T=1200^{\circ} \mathrm{C}$ follows next. For small samples of free-form structures and 3D lattices, the results are shown in S.Fig. 2. Structural elements having a diameter less than 3-5 $\mu \mathrm{m}$ succumb to melting or at least softening followed by surface tension induced deformation (rounding). This can be advantages for indirect morphing into new shapes with improved surface smoothness ${ }^{26,27}$.

For structures with larger features, such as scaffolds examined in this study, the geometry is maintained as shown in S.Fig. 3. The S.Fig. 3(a) shows that small topological features such as textures on the vertical columns present in the initial glass-phase structures disappeared in the ceramic state. Also, the right angles horizontal beams become rounded. However, the pattern structure was maintained

\footnotetext{
${ }^{1 *}$ For reproducibility purposes we note that the STL model used here is publicly available and was retrieved from https://www.thingiverse.com on Sep 20, 2017 under the name "Ballet Dancer 03" as published on Oct 11, 2016.
} 
after heat treatment as shown in the SEM micro-graphs S.Fig. 3. Some random cracks can be observed in the annealed structures, however, since the structures were not attached to the substrate and had to be handled multiple times when transferring them mechanically from substrate to substrate (glass substrate - fabrication, corundum substrate - furnace, adhesive carbon tape - SEM imaging) we cannot exclude the cause of cracks being due to a mechanical strain from handling.

We summarize that for the particular case of the DLW structures having feature sizes smaller than $3 \mu \mathrm{m}$, they are strongly affected by the thermal re-flow process, but the structures with features larger than approximately $10 \mu \mathrm{m}$ rescaled by maintaining the original outline shape.

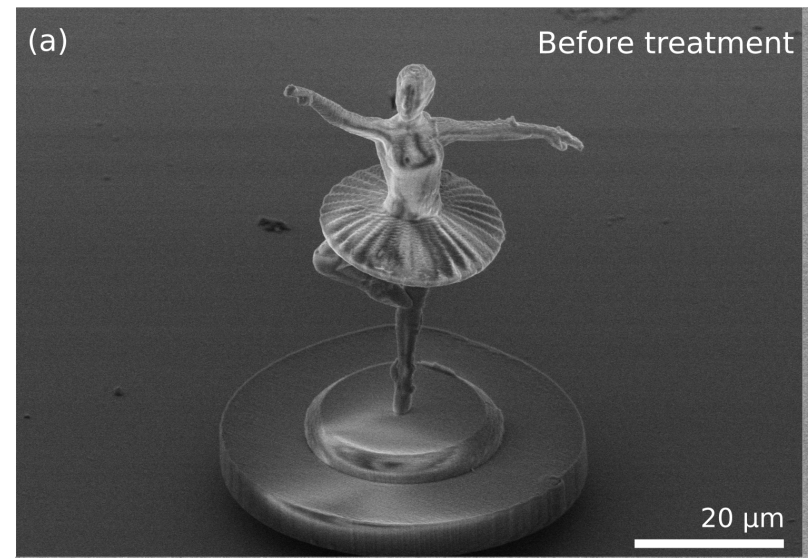

(b)

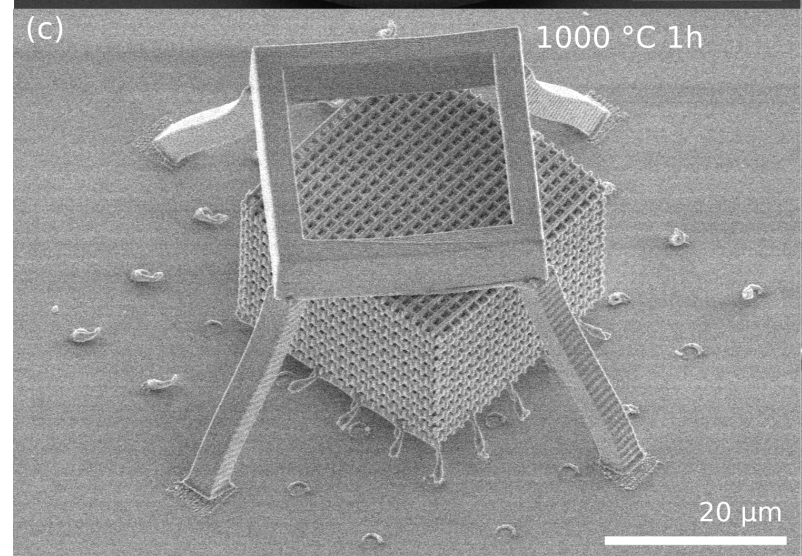

(d)
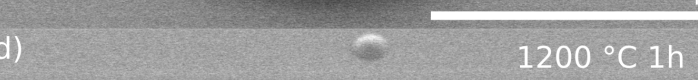

$200^{\circ} \mathrm{C} 1 \mathrm{~h}$

Figure 2. SEM micrographs of a fine-feature ballerina dancer (free-form) sculpture*: untreated (a) and thermally annealed ceramic structure (b). Also, a periodic 3D grating with a cage is shown in a glassy phase (c) and in a ceramic phase (d). 

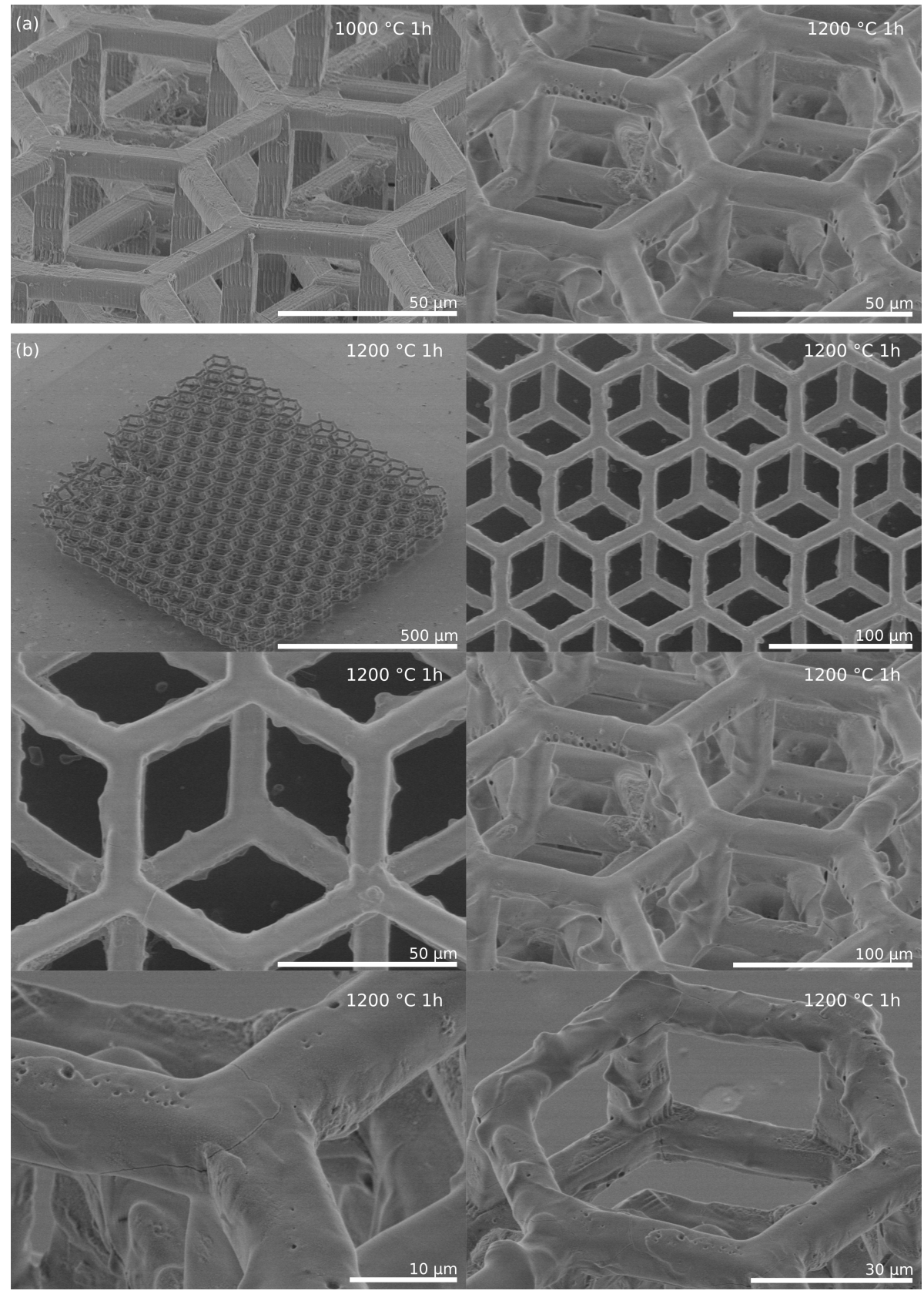

Figure 3. SEM micrographs of large areas scaffold after heat treatment at different magnifications; temperature and duration of annealing are indicated on the SEM images. 